\title{
Analysis of the Finite-Temperature Phase Diagram of the Spinless Falicov-Kimball Model
}

\author{
R. LEMAŃSKI \\ Institute of Low Temperature and Structure Research, Polish Academy of Sciences, \\ Okólna 2, 50-422 Wrocław, Poland
}

\begin{abstract}
The finite-temperature phase diagram of the spinless Falicov-Kimball model on the Bethe lattice is analyzed using the dynamic mean field theory. Comparing the temperature-dependent density of states at various phases we detected a difference between two phases of the ordered insulator (OI-X and OI-Y) whose stability areas in the phase diagram are separated by the stability area of the ordered conductor. It appears that the difference between OI-X and OI-Y phases is due to the band inversion, consisting in a reversal of those subbands, that lie just above and below the Fermi level and are derived only from one or the other sublattice $(+$ or - ).
\end{abstract}

DOI: 10.12693/APhysPolA.130.577

PACS/topics: 71.10.Fd, 71.20.--b, 71.45.Lr

\section{Introduction}

Correlated electron systems still no cease to amaze physicists due to their intriguing properties. Even the simplest models of these systems show remarkable features. One of these models is the spinless FalicovKimball model (FKM) [1]. It describes itinerant electrons interacting with localized ions via the local (on-site) Coulomb coupling $U$.

Here we consider two types of the ions $A$ and $B$, whose densities $\rho_{A}$ and $\rho_{\mathrm{B}}$, respectively, are equal to $1 / 2\left(\rho_{A}=\right.$ $\left.\rho_{\mathrm{B}}=1 / 2\right)$ and spinless electrons, whose density $\rho_{d}$ is also equal to $1 / 2$ (half-filling).

The Hamiltonian of the FKM we use is (see Refs. $[2,3])$ :

$$
H=t \sum_{\langle m, n\rangle} d_{m}^{+} d_{n}+U \sum_{m} w_{m} n_{m}^{d}
$$

where $\langle m, n\rangle$ means the nearest neighbor lattice sites $m$ and $n, d_{m}\left(d_{m}^{+}\right)$is an annihilation(creation) operator of itinerant electrons, whereas $n_{m}^{d}$ is their occupation number operator. The quantity $w_{m}$ is equal to $1 / 2(-1 / 2)$ for the lattice site occupied by the ion $A(B)$, therefore the Coulomb-type on-site interaction between itinerant electrons and the ions amounts $U / 2(-U / 2)$. The hopping electron amplitude $t$ we henceforth set equal to one, so we measure all energies in units of $t$.

The ground state of the system has the checkerboardtype structure composed of two interpenetrating sublattices + and - , each of which is occupied only by one type of ion: the sublattice + by the ions $A$ and the sublattice - by the ions $B$, respectively [3]. Consequently, the density $\rho_{A}^{+}\left(\rho_{B}^{-}\right)$of ions $A(B)$ on the sublattice + $(-)$ is equal to $1\left(\rho_{A}^{+}=\rho_{B}^{-}=1\right)$, whereas the density $\rho_{B}^{+}$ $\left(\rho_{A}^{-}\right)$of ions $B(A)$ on the sublattice $+(-)$ is equal to $0\left(\rho_{B}^{+}=\rho_{A}^{-}=0\right)$.

With an increase of temperature the densities $\rho_{A}^{+}, \rho_{B}^{-}$ $\left(\rho_{A}^{+}=\rho_{B}^{-}\right)$diminish below 1 , while $\rho_{B}^{+}, \rho_{A}^{-}\left(\rho_{B}^{+}=\rho_{A}^{-}\right)$ increase above 0 and in the disordered phase all these densities are equal to $1 / 2$. Therefore the quantity $d=$ $\rho_{A}^{+}-\rho_{A}^{-}=\rho_{B}^{-}-\rho_{B}^{+}$is chosen to be the order parameter.

In Ref. [3] it is shown that in addition to the orderdisorder phase transition, the model (1) describes also the metal-insulator transformation. This transformation results from the temperature dependence of the density of states (DOS) and possible changes in the DOS value at the Fermi level, which may be zero (in which case we are dealing with an insulator) or take a positive value (then we are dealing with a conductor).

The $U-T$ phase diagram of the model was obtained in Ref. [3] rigorously within the dynamic mean field theory (DMFT) formalism (see Fig. 1) and its main message was to show that the system could be a conductor not only in the disordered phase, but also in the ordered phase, and for a particular $U=U_{c r}$, even down to $T=0$. In this phase diagram, there have been found the stability areas of four phases: the ordered insulator (OI), the ordered conductor (OC), the disordered insulator (DI), and the disordered conductor (DC). In the ordered phases OC and OI, the charge-density-wave order of the checkerboard type is present, whereas the disordered phases DC and DI are homogeneous. On the other hand, DOS at the Fermi level $\rho\left(E_{F}\right)=0$ in the insulating phases OI and DI, whereas $\rho\left(E_{F}\right)>0$ in the conducting phases OC and DC.

Surprisingly, it turned out that the stability area of OI phase is divided into two separate parts OI-X nad OI$\mathrm{Y}$, between which the stability area of OC phase occurs. Moreover, the stability areas of these three phases (OI$\mathrm{X}, \mathrm{OI}-\mathrm{Y}$, and $\mathrm{OC}$ ) contact each other only at one point, at $T=0$ and $U=U_{c r}=\sqrt{2}$, which corresponds to the quantum critical point. On the other hand, on the phase diagram of this system that was before obtained numerically in Ref. [4] the stability area of the OI phase was not separated into two distinct areas and the quantum critical point was not detected.

Since a qualitative difference between the two phases is an important prerequisite for an existence of the quan- 
tum critical point $T=0$, our goal here is to extend the research initiated in Ref. [3] by analyzing the phase diagram and focusing on finding a difference between the phases OI-X and OI-Y.

\section{Method of calculation}

Our analysis of the phase diagram is based on an examination of the temperature-dependent DOS distributions $\sigma(U, T ; E)$ in different phases. Here $\sigma(U, T ; E)$ is derived for the Bethe lattice in the limit of large dimensions using the exact formulae for the Green functions within the DMFT formalism. The formalism was derived and described in Refs. $[5,6]$, where it was applied to the limits of small and large $U$ values only and subsequently it was used in Ref. [3], where intermediate couplings were examined.

The method is based on the following two exact analytical formulae for the Green functions $G^{+}(z)$ and $G^{-}(z)$, referring to the sublattice + and - , respectively $[5,6]$ :

$$
\begin{aligned}
& G^{+}(z)=\frac{z+\frac{1}{2} U d-G^{-}(z)}{\left[z+\frac{1}{2} U-G^{-}(z)\right]\left[z-\frac{1}{2} U-G^{-}(z)\right]} \\
& G^{-}(z)=\frac{z-\frac{1}{2} U d-G^{+}(z)}{\left[z+\frac{1}{2} U-G^{+}(z)\right]\left[z-\frac{1}{2} U-G^{+}(z)\right]},
\end{aligned}
$$

where $d$ is the order parameter.

After finding self-consistent solutions of Eqs. (2) one gets the expressions for electron $\operatorname{DOS} \sigma^{+}(U, d ; E)$ and $\sigma^{-}(U, d ; E)$ corresponding to the sublattice + and - , respectively from the standard formulae

$$
\begin{aligned}
& \sigma^{+}(U, d ; E)=-\frac{1}{\pi} \operatorname{Im} G^{+}(U, d ; E+\mathrm{i} 0), \\
& \sigma^{-}(U, d ; E)=-\frac{1}{\pi} \operatorname{Im} G^{-}(U, d ; E+\mathrm{i} 0),
\end{aligned}
$$

and hence the total DOS

$$
\sigma(U, d ; E)=\left[\sigma^{+}(U, d ; E)+\sigma^{-}(U, d ; E)\right] / 2 .
$$

The functions $G^{+}(z)$ and $G^{-}(z)$, then also $\sigma^{+}(U, d ; E)$ and $\sigma^{-}(U, d ; E)$, are not explicitly dependent on $T$, so their temperature dependence comes out entirely from the temperature dependence of $d=d(U, T)$. Consequently, both the order parameter $d(U, T)$ and the DOS distributions $\sigma^{+}(U, d ; E)$ and $\sigma^{-}(U, d ; E)$ are determined self-consistently from the following procedure. First we determine the free energy $F(U, d, T)$ from the formula $[7,8]$ :

$$
\begin{aligned}
& F(U, d, T)= \\
& \quad T \int_{-\infty}^{\infty} \mathrm{d} E \sigma(U, d ; E) \ln \frac{1}{1+\exp \left(-E / k_{\mathrm{B}} T\right)}-T S_{\text {ions }},
\end{aligned}
$$

where the ions entropy $S_{\text {ions }}$ is given by

$$
S_{\text {ions }}=-\frac{1+d}{2} \ln \frac{1+d}{2}-\frac{1-d}{2} \ln \frac{1-d}{2} .
$$

By minimizing $F(U, d, T)$ over $d$ we find the order parameter $d(U ; T)$ and then $\sigma^{+}(U, d ; E)$ and $\sigma^{-}(U, d ; E)$. The entire procedure is described in more detail in Ref. [3].

\section{Results}

From our calculations no qualitative difference between the total DOS $\sigma(U, d ; E)$ in OI-X and OI-Y was found.
Indeed, in both these phases $\sigma(U, d ; E)$ is symmetric with respect to the Fermi level $E_{\mathrm{F}}=0$. At $T=0$, when the order parameter $d=1$, it consists of two bands separated by the energy gap equal to $U$. On the other hand, at temperatures greater than zero (then $d<1$ ), two additional subbands symmetrically distributed with respect to $E_{\mathrm{F}}=0$ are formed inside the energy gap. Merely for $U=U_{c r}=\sqrt{2}$ only the one subband, whose center lies exactly at the Fermi level $E_{\mathrm{F}}=0$, instead of two sub-bands, is formed. Thus, for both $U<\sqrt{2}$ and for $U>\sqrt{2}$, i.e. for the OI-X and OI-Y phase, the total DOS looks similar and for $d<1$ consists of two main bands and two sub-bands, which arise inside the energy gap.

As it turns out, this symmetry applies only to the total (resultant) DOS $\sigma(U, d ; E)$. However, $\sigma^{+}(U, d ; E)$ and $\sigma^{-}(U, d ; E)$, when considered separately, are not symmetric. This is clearly seen by watching the DOS distributions inside the principal energy gap. Indeed, for $U<\sqrt{2}, \sigma^{+}(U, d ; E)$ has lower weight in the subband lying below $E_{\mathrm{F}}=0$ than in the subband situated above $E_{\mathrm{F}}=0$, whereas $\sigma^{-}(U, d ; E)$ has lower weight in the subband lying above $E_{\mathrm{F}}=0$ than in the subband situated below $E_{\mathrm{F}}=0$. Conversely, when $U>\sqrt{2}, \sigma^{+}(U, d ; E)$ has higher weight in the subband lying below $E_{\mathrm{F}}=0$ than in the subband situated above $E_{\mathrm{F}}=0$, whereas $\sigma^{-}(U, d ; E)$ has higher weight in the subband lying above $E_{\mathrm{F}}=0$ than in the subband situated below $E_{\mathrm{F}}=0$. The kind of asymmetry described here does not apply to the major bands (upper and lower). Namely, $\sigma^{+}(U, d ; E)$ has lower weight in its lower main band than in the upper main band for any value of $U>0$. Likewise, for any value of $U>0, \sigma^{-}(U, d ; E)$ has greater weight in its lower main band than in the upper main band.

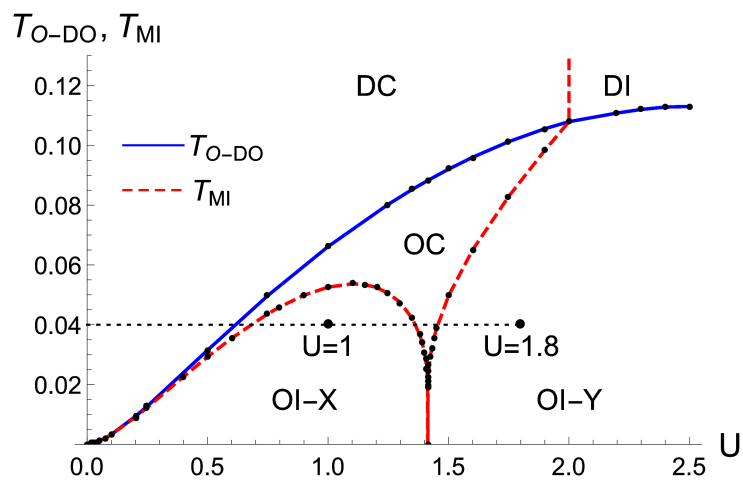

Fig. 1. Phase diagram of the spinless FKM at half filling in the plane $(U, T)$. The points placed on the dotted line at $T=0.04$ indicate those phases for which the DOS is presented in Figs. 3 and 4.

In Fig. 1 we display the $U-T$ phase diagram of the model. In this diagram the stability area of the OC phase tapers to a line when $U=\sqrt{2}$ and $T \rightarrow 0$. For this reason, we constructed in addition the $d-U$ phase diagram, where the temperature $T$ was replaced by the 


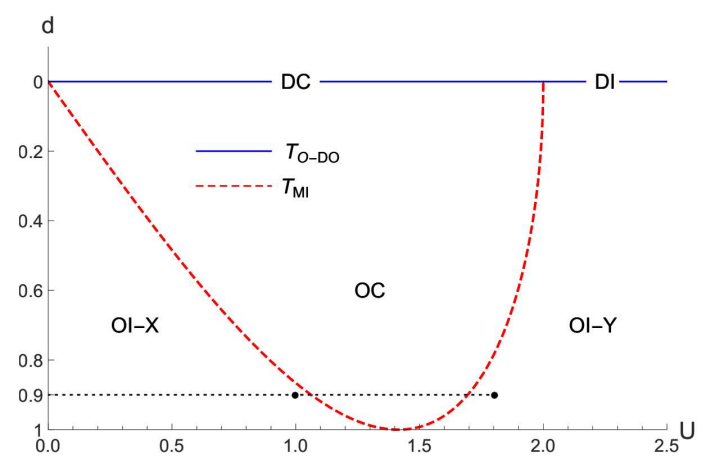

Fig. 2. The phase diagram of the spinless FKM at half filling in the plane $(d, T)$. Points placed on the dotted line at $d=0.9$ indicate those phase for which DOS is shown in Figs. 3 and 4.

order parameter $d$ (see Fig. 2). Then, it turned out that for $d<1$ the stability range of the OC phase covers a wide area, which tapers to a critical point only in the limit $d \rightarrow 1$. This makes it easier to analyze the behavior of the system in the vicinity of the quantum critical point, in particular in the OC phase.

The DOS distributions for $T=0.04$ at two selected values of $U=1,1.8$ are shown in Figs. 3 and 4. One can see there that in accordance with the description given above, the lower sub-band corresponding to the DOS of the sublattice + has less weight than the upper sub-band when $U<\sqrt{2}$, while it has more weight when $U>\sqrt{2}$.

Since for a fixed value of $T$ the DOS weights of sub-bands lying inside the energy gap significantly decrease with an increase of $U$, and already for $U=1.8$ are very small (barely visible) compared with the weights DOS of the main bands (see Fig. 3), we display separately in Fig. 4 a part of the DOS in the vicinity of the Fermi level. We also present the DOS distributions at a fixed value of the order parameter $d=0.9$ in Fig. 5 , where one can clearly note its asymmetry on the sublattices + and - .

\section{Conclusions}

Our analysis of the DOS revealed some unexpected properties of its distribution on individual sublattices. It turned out that in both sublattices + and - , a capacity of the main lower band and a capacity of the lower subband, lying inside the main energy gap just below the Fermi level, change in the opposite direction when $U$ changes. This behavior resembles the phenomenon of inversion of energy bands, that is intensively discussed nowadays in a context of the topological insulators. There, however, the inversion concerns to the whole system, while in our case it concerns only to individual sublattices. Therefore, it is not clear what physical effects could be influenced by the inversions related to sublattices only. Anyway, since $U$ can be changed by applying an external pressure to the system (in fact, the relation $U / t$ changes then), it can be assumed that this kind of inversion of bands can happen.

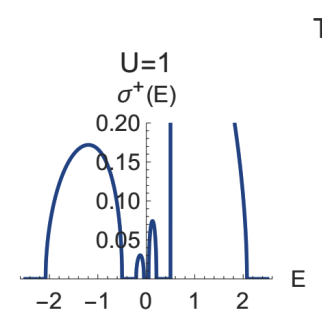

$\mathrm{T}=0.04$
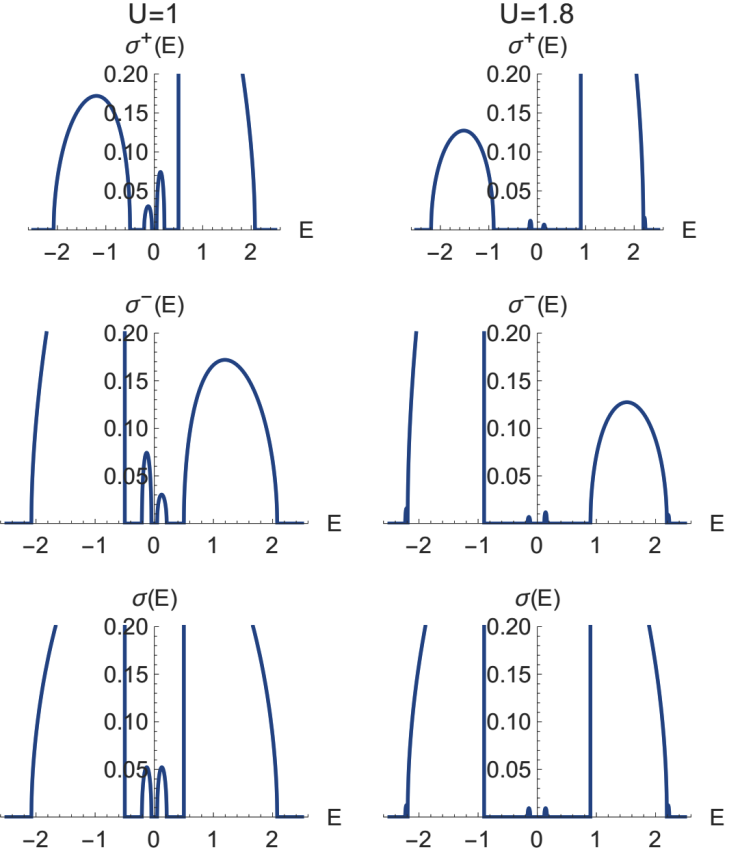

Fig. 3. The DOS of the spinless FKM at $T=0.04$. The left column corresponds to the OI-X phase at $U=1$ and the right column corresponds of the OI-Y phase at $U=1.8$. The first row - the DOS of the sublattice +, second row - the DOS of the sublattice -, third row - the total DOS.
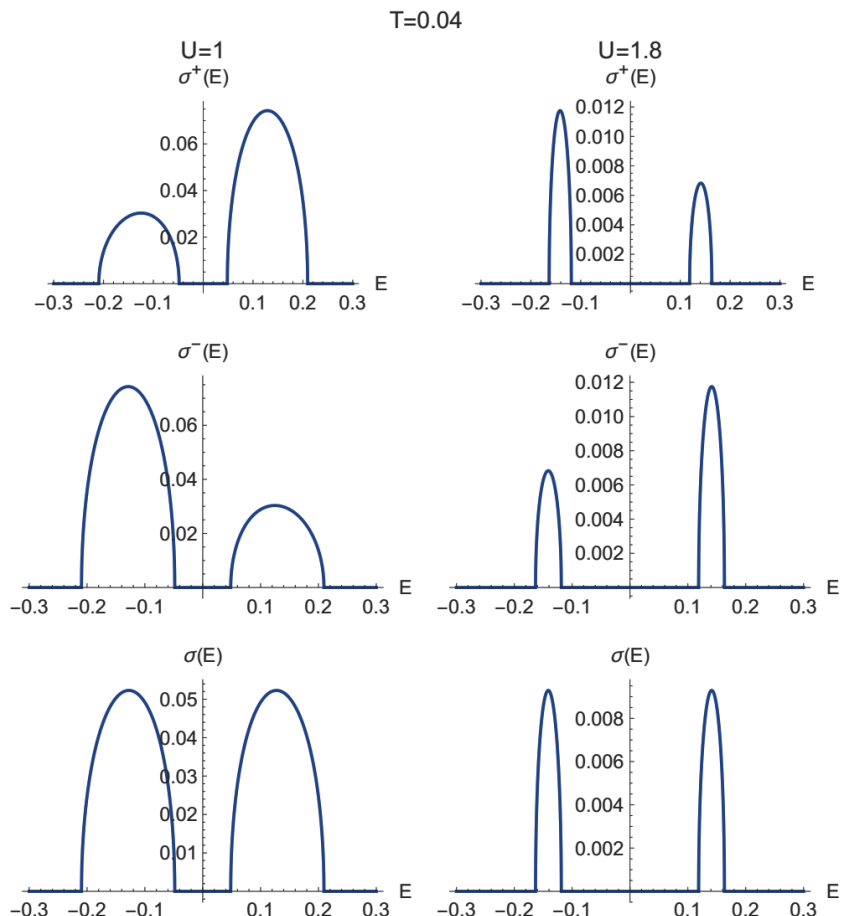

Fig. 4. A part of the DOS of the spinless FKM at $T=$ 0.04 in the vicinity of the Fermi level. The description is the same as in the caption to Fig. 3 . 


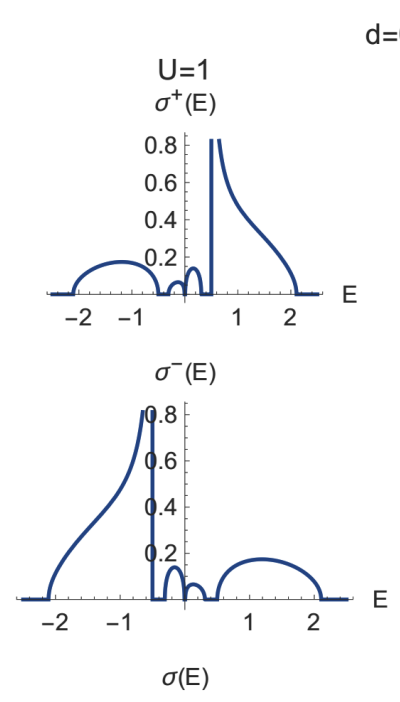

$d=0.9$
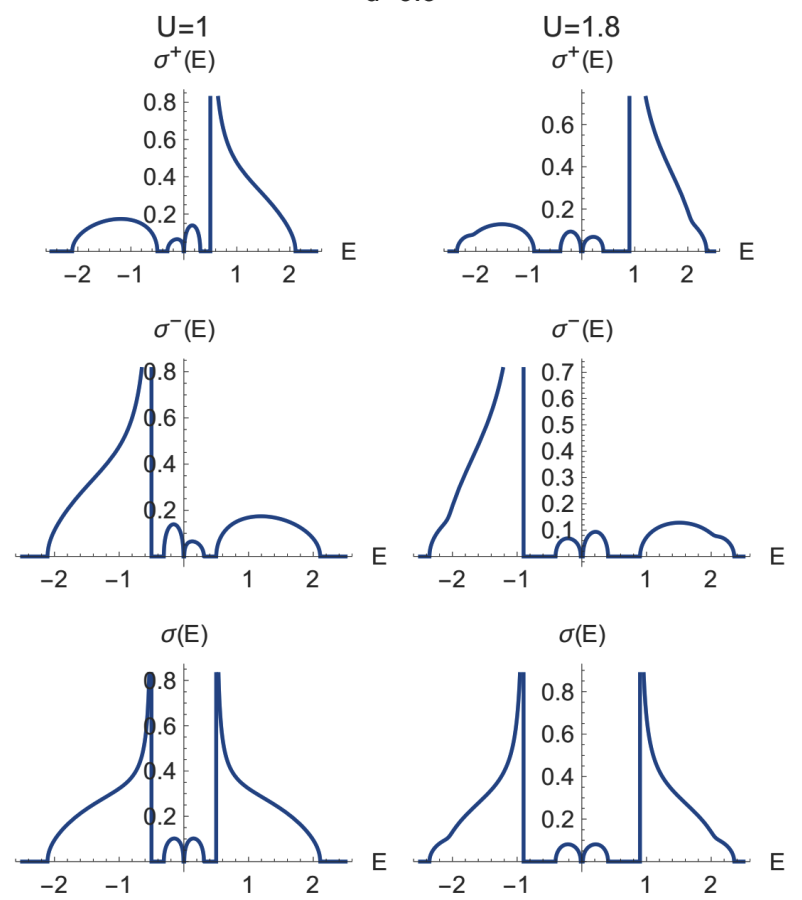

Fig. 5. The DOS of the spinless FKM for the order parameter $d=0.9$. The left column corresponds to the OI-X phase at $U=1$ and the right column corresponds of the OI-Y phase at $U=1.8$. The first row - the DOS of the sublattice + , second row - the DOS of the sublattice -, third row - the total DOS.

That is, if a change of $U$ causes an increase (decrease) of the number of available electronic states in the main lower band, then the number of available electronic states in the lower subband decreases (increases). Clearly, since the capacity of the sub-band is smaller than the capacity of the main band, a resultant change of the number of available states below the Fermi level for a given sublattice is determined by the change in the main band.

It also turns out that with increasing $U$ sub-bands lying inside the main energy gap approach each other when $U<U_{c r}$ and go apart from each other when $U>U_{c r}$. Then, of course, for $U=U_{c r}$, these two sub-bands become identical, so they merge into one sub-band with its center at $E_{\mathrm{F}}=0$. The consequence of these properties is that in the OI-X phase, the lower (upper) sub-band on the sublattice $+(-)$ has a capacity smaller than the upper (lower) sub-band. The opposite situation occurs in the OI-Y phase, since then the lower (upper) sub-band on the sublattice $+(-)$ has a capacity higher than the upper (lower) sub-band.
Thus, due to the DOS asymmetry at individual sublattices + and - we can determine the difference between the phases of OI-X and OI-Y. On the sublattice + in the OI-X phase there are more electronic states in the bottom subband than in the upper one (of subbands lying inside the band gap) and in the OI-Y phase there are more electron states in the upper subband than in the bottom one.

The obtained here results relate to the specific case of the Bethe lattice (which made it possible to get rigorous outcomes), but taking into account recent research of the DOS performed using the Monte Carlo method on the two-dimensional square lattice $[9,10]$ and the DMFT method on the high dimension hypercubic lattice $[4,11]$, one can expect that they are universal. Another issue is to determine an influence of the found here features of the DOS on physical properties of the system. In particular, it is about its properties in the vicinity of the critical point $U=\sqrt{2}, T=0$. But this subject requires further studies.

\section{Acknowledgments}

I am grateful to Dariusz Kaczorowski for the suggestion to study issues presented in this work.

\section{References}

[1] L.M. Falicov, J.C. Kimball, Phys. Rev. Lett. 22, 997 (1969).

[2] J.K. Freericks, R. Lemański, Phys. Rev. B 61, 13438 (2000).

[3] R. Lemański, K. Ziegler, Phys. Rev. B 89, 075104 (2014).

[4] S.R. Hassan, H.R. Krishnamurthy, Phys. Rev. B 76, 205109 (2007).

[5] P.G.J. van Dongen, Phys. Rev. B 45, 2267 (1992).

[6] P.G.J. van Dongen, C. Leinung, Ann. Phys. 6, 45 (1997).

[7] J.K. Freericks, Ch. Gruber, N. Macris, Phys. Rev. B 60, 1617 (1999).

[8] A.M. Shvaika, J.K. Freericks, Phys. Rev. B 67, 153103 (2003).

[9] H. Čenčariková, M. Žonda, P. Farkašovský, Solid State Commun. , 149, 1997 (2009).

[10] M. Maśka, K. Czajka, Phys. Rev. B 74, 035109 (2006).

[11] O.P. Matveev, A.M. Shvaika, J.K. Freericks, Phys. Rev. B 77, 035102 (2008). 